\title{
Stable minimal surfaces of finite total curvature
}

\author{
Claudio Arezzo ${ }^{1}$, Mario J. Micallef and Gian Pietro Pirola ${ }^{2}$
}

\section{Introduction.}

It is well known that holomorphic submanifolds of Kähler manifolds minimize volume in their homology class. As pointed out in [1], the converse can be expected to hold only when the ambient manifold satisfies some extra conditions, usually in the form of a non negativity assumption on the curvature. The special situation of area minimizing surfaces in the Euclidean space $E^{n}$ of dimension $n$ is particularly worth studying because of its connections with the Bernstein Theorem (see, for example, [8]) and, possibly, with regularity theory. This is the problem mentioned in Yau's Open Problems list (see [14]), and it is the one we want to study in this paper.

We start by recalling two of the main theorems in [8]:

1. a stable minimal isometric immersion of a complete oriented parabolic surface into $E^{4}$ is holomorphic w.r.t. some orthogonal complex structure,

2. a stable isometric minimal immersion of a complete oriented surface of finite total curvature and genus zero into $E^{2 n}$ is holomorphic w.r.t. some orthogonal complex structure on $E^{2 n}$.

By a well known result of Osserman ([12]), every complete oriented minimal surface $\Sigma^{*}$ of finite total curvature is conformally equivalent to a closed Riemann surface $\Sigma$ punctured at finitely many points and hence parabolic. The result in (1) therefore applies to oriented stable minimal surfaces of finite total curvature and of arbitrary genus in $E^{4}$.

\footnotetext{
${ }^{1}$ Research partially supported by European Community Grant TRM N.ERBFMBICT 950079.

${ }^{2}$ Partially supported by $40 \%$ project Geometria Algebrica M.U.R.S.T., by G.N.S.A.G.A. (C.N.R.) Italy and by European project Sci. " Geom. of Alg. Varieties".
} 
Arezzo-Micallef ([1]) have recently produced many examples of stable closed minimal surfaces of genus $g \geq 4$ in flat tori of (real) dimension $2 g$ and, for $g>\frac{5+2 k+\sqrt{1+24 k}}{2}$, into tori of dimension $2(g-k)$ with $k=1,2,3$.

Inspired by their work we have tried to adapt this strategy to the case of complete oriented minimal surfaces of non zero genus and of finite total curvature in Euclidean space.

In particular we prove:

Theorem 1. Given any closed Riemann surface $\Sigma$ of genus 2 and a Weierstrass point $p \in \Sigma$, and any $n \geq 11$, there exists a stable conformal minimal embedding of $\Sigma \backslash\{p\}$ into $E^{2 n}$, of finite total curvature $\mathcal{K}$ equal to $-2 \pi(n+5)$, which is not holomorphic w.r.t. any complex structure compatible with the euclidean metric.

As in [1], the strategy of the construction is to consider a holomorphic embedding (which is, by Wirtinger's Inequality, a stable minimal immersion) and to deform it, destroying holomorphicity but preserving all other riemannian properties. The main technical difficulty in making this program to work is to get control on the space of Jacobi fields of a holomorpihc curve seen as a minimal surface. In particular, a necessary condition for our strategy to construct families of stable minimal surfaces is that the starting holomorphic curve has only translational bounded Jacobi fields.

In Theorem 2 we prove that the existence of such affine curves is equivalent to the fact that, having set $W$ the space of exact meromorphic 1-forms on $\Sigma \backslash\{p\}$ with a pole of order at most $m$ at $p$, the cup-product map

$$
\tilde{\phi}: W \otimes H^{0}(K) \rightarrow H^{0}\left(K^{2} \otimes[m p]\right)
$$

is surjective.

Given two line bundles $L$ and $M$ over a Riemann surface and a subspace $V \subset H^{0}(L)$, the surjectivity of multiplicative maps of the form

$$
V \otimes H^{0}(M) \rightarrow H^{0}(L \otimes M)
$$

is a very classical subject in the theory of Algebraic curves, being related, for example, to the projective normality of linear series (see, e.g., [3], [4] and [5]).

Typically this theory deals with the case of a generic subspace $V$ of $H^{0}(L)$ of a fixed dimension, while in our case we need $W$ to be a fixed (and in fact a very special!) subspace of $H^{0}(K \otimes[m p])$. Therefore we need to use completely different techniques, similar to the ones used in ([10]). 
We believe that our method can be used also in the case of surfaces of genus greater than 2 with more than one puncture, so as to improve the estimate on the dimension of the ambient Euclidean space (and maybe also on the total curvature).

We observe in Section 2 that our method cannot be applied to study immersions in $E^{6}$ for any genus since any holomorphic map into $\mathbb{C}^{3}$ has non translational bounded Jacobi fields corresponding to the failure of the surjectivity of the cup-product map mentioned above. We leave this problem for further investigation.

Acknowledgments. The first author is indebted to Rick Schoen for providing warm hospitality at Stanford University during the preparation of this paper.

\section{Deformation of holomorphic curves of finite total curvature to stable minimal surfaces.}

Let $\psi: \Sigma^{*} \rightarrow E^{n}$ be a complete minimal immersion of finite total curvature. We know from [12] that $\Sigma^{*}$ is conformally equivalent to a closed Riemann surface $\Sigma$ punctured at finitely many points $\left\{p_{1}, \ldots, p_{k}\right\}$. Furthermore, if $\operatorname{Gr}(2,2 n) \subset \mathbb{C} P^{n-1}$ denotes the Grassmannian of oriented two planes in $E^{2 n}$ then the Gauss map $G: \Sigma^{*} \rightarrow G r(2,2 n)$ extends to a holomorphic map $\bar{G}: \Sigma \rightarrow G r(2,2 n)$. We can therefore pull-back via $\bar{G}$ the tautological 2plane bundle $\gamma$ and its orthogonal complement $2 n-2$-bundle $\gamma^{\perp}$, obtaining two bundles over $\Sigma, \overline{\tau_{\psi}}$ and $\overline{\nu_{\psi}}$ respectively. By definition these bundles have the following properties:

1. $\overline{\tau_{\psi}} \oplus \overline{\nu_{\psi}}$ is the trivial $2 n$ bundle over $\Sigma$;

2. ${\overline{\tau_{\psi}}}_{\Sigma_{\Sigma^{*}}}$ is the tangent bundle to $\psi\left(\Sigma^{*}\right)$, and

3. $\bar{\nu}_{\Sigma_{\Sigma^{*}}}$ is the normal bundle to $\psi\left(\Sigma^{*}\right)$.

In the Theorem below we shall need to consider $\overline{\tau_{\psi}}$ as a complex line bundle whose fibres on $\Sigma^{*}$ are locally spanned by $\psi_{z}$ where $z$ is a local complex coordinate. At each $p_{i}, \psi_{z}$ has a pole of order $m_{i}$, i.e., $\psi_{z}=$ $z^{-m_{i}} v(z)$ where $v$ is holomorphic and $v(0) \neq 0$.

Let $[D]$ be the line bundle corresponding to the divisor $D$.

Then $\overline{\tau_{\psi}}=T \Sigma \otimes\left[-\sum_{i=1}^{k} m_{i} p_{i}\right]$ and therefore ${\overline{\tau_{\psi}}}^{-1}=K \otimes\left[\sum_{i=1}^{k} m_{i} p_{i}\right]$, where $K$ is the canonical bundle of $\Sigma$. Let $s$ be a holomorphic section of [ $\sum_{i=1}^{k} m_{i} p_{i}$ ] with a zero of order $m_{i}$ at $p_{i}$ ( $s$ is unique up to multiplication by a non zero complex number). 
Then, for each $j \in\{1, \ldots, n\}, \partial \psi^{j} \otimes s \in H^{0}\left({\overline{\tau_{\psi}}}^{-1}\right)$.

Theorem 2. Let $\psi: \Sigma^{*} \rightarrow \mathbb{C}^{n}$ be a complete holomorphic immersion of finite total curvature. Consider the map $\mu^{*}: H^{0}\left(\mathbb{C}^{n} \otimes K\right) \rightarrow H^{0}\left({\overline{\tau_{\psi}}}^{-1} \otimes K\right)$ given by

$$
\left(\theta_{1}, \ldots, \theta_{n}\right) \rightarrow\left(\left(d \psi^{1} \otimes s\right) \cdot \theta_{1}+\cdots+\left(d \psi^{n} \otimes s\right) \cdot \theta_{n}\right),
$$

where $s$ is a holomorphic section of $\left[\sum_{i=1}^{k} m_{i} p_{i}\right]$ as defined above, and . denotes the cup-product mapping between $H^{0}\left(K \otimes\left[\sum_{i=1}^{k} m_{i} p_{i}\right]\right)$ and $H^{0}(K)$. If

1. the differentials $d \psi^{1}, \ldots, d \psi^{n}$ satisfy a non trivial quadratic equation of the form $\sum_{j=1}^{r}\left(d \psi^{j}\right)^{2}=0$ and,

2. $\mu^{*}$ is surjective,

then there exists a 1-parameter family $\psi_{t}: \Sigma^{*} \rightarrow E^{2 n}$ of stable minimal immersions such that $\psi_{0}=\psi$, the total curvature of $\psi_{t}$ is constantly equal to the total curvature of $\psi$ and, for $t \neq 0, \psi_{t}$ is not holomorphic w.r.t. any complex structure compatible with the Euclidean metric.

Proof. Viewing $\mathbb{C}^{n}$ as $\left(\mathbb{R}^{2 n}, J_{0}\right)$ (where we denote by $J_{0}$ the standard complex structure) we can write

$$
\psi(q)=\operatorname{Re}\left(\int_{q_{0}}^{q} d \psi^{1}, \ldots, d \psi^{n}, i d \psi^{1}, \ldots, i d \psi^{n}\right) .
$$

Let us define

$$
\begin{aligned}
\psi_{t}(q)=\operatorname{Re}\left(\int_{q_{0}}^{q}(1+t) d \psi^{1}, \ldots,(1+t) d \psi^{r}, d \psi^{r+1}, \ldots,\right. & \\
& \left.d \psi^{n}, i d \psi^{1}, \ldots, i d \psi^{n}\right) .
\end{aligned}
$$

Clearly $\psi_{0}=\psi$ and by (2) and the Weierstrass Representation Theorem ([11]) $\psi_{t}$ is a 1-parameter family of conformal minimal immersions.

$\psi_{t}$ has finite total curvature $\mathcal{K}$ : indeed,

$$
2 \pi\left(2-2 g-\sum_{j=1}^{k} m_{j}\right)=2 \pi c_{1}\left(\overline{\tau_{\psi}}\right)=2 \pi \operatorname{deg}(\bar{G})=\mathcal{K}
$$


which does not depend on $t$. Formula (2.1) is known as the Jorge-Meeks formula ([7]).

The next two lemmas conclude the proof of Theorem 1:

Lemma 2.1. There exists $t_{0}>0$ s.t. for any $|t|<t_{0}, \psi_{t}$ is stable.

Proof. Fischer-Colbrie ([2]) and Nayatani ([9]) have proved that the Jacobi operator $J^{\psi}$ on $\Sigma^{*}$ extends to an operator $\overline{J^{\psi}}$ on $\Sigma$ with the same index as that of $J^{\psi}$. Let us remark (even though we will not use this relation) that a simple potential theoretic argument shows that every smooth bounded Jacobi field on $\psi\left(\Sigma^{*}\right)$ extends to a smooth section of $\overline{\nu_{\psi}}$, i.e.,

$$
\operatorname{ker}\left(\overline{J^{\psi}}\right)=\left\{\text { bounded Jacobi fields on } \psi\left(\Sigma^{*}\right)\right\} .
$$

Simons's Theorem 3.5.1 in [13] applies to $\overline{J^{\psi_{0}}}$ showing that $\operatorname{ker}\left(\overline{J_{0}}\right)=$ $H^{0}\left(\overline{\nu_{\psi_{0}}}\right)$.

We now claim that

$$
H^{0}\left(\overline{\nu_{\psi_{0}}}\right) \simeq \mathbb{C}^{n}
$$

i.e., along $\psi_{0}$ there are only translational Jacobi fields: indeed, by definition, the sequence

$$
0 \rightarrow \overline{\tau_{\psi_{0}}} \rightarrow \mathbb{C}^{n} \rightarrow \overline{\nu_{\psi_{0}}} \rightarrow 0
$$

and the associated long exact sequence

$$
0 \rightarrow H^{0}\left(\mathbb{C}^{n}\right) \stackrel{\Xi}{\rightarrow} H^{0}\left(\overline{\nu_{\psi_{0}}}\right) \stackrel{\Phi}{\rightarrow} H^{1}\left(\overline{\tau_{\psi_{0}}}\right) \stackrel{\mu}{\rightarrow} H^{1}\left(\mathbb{C}^{n}\right) \rightarrow \cdots
$$

are exact.

Since these sequences are exact we have immediately $h^{0}\left(\overline{\nu_{\psi_{0}}}\right) \geq n$. Geometrically this is just the fact that $H^{0}\left(\mathbb{C}^{n}\right)$ corresponds to the translations in the euclidean space which, of course, induce bounded Jacobi fields on the surface.

By Kodaira-Serre duality

$$
H^{1}\left(\overline{\tau_{\psi_{0}}}\right)=\left(H^{0}\left(K \otimes{\overline{\tau_{\psi_{0}}}}^{-1}\right)\right)^{*} \text { and } H^{1}\left(\mathbb{C}^{n}\right)=\left(H^{0}\left(\mathbb{C}^{n} \otimes K\right)\right)^{*} .
$$

We observe that if $\mu$ is injective then $\Phi=0$, in which case $\operatorname{ker}(\Phi)=$ $H^{0}\left(\overline{\nu_{\psi_{0}}}\right)=\operatorname{im}(\Xi) \simeq \mathbb{C}^{n}$. Now $\mu$ is injective iff the dual map, $\mu: H^{0}\left(\mathbb{C}^{n} \otimes\right.$ $K) \rightarrow H^{0}\left(K \otimes \bar{\tau}_{\psi_{0}}-1\right)$, surjective. But this is precisely condition (2) (see ([1]) for details, if necessary).

By Wirtinger's Inequality, $0=\operatorname{index}\left(J^{\psi_{0}}\right)=\operatorname{index}\left(\overline{J^{\psi_{0}}}\right)$. Moreover $\overline{\nu_{\psi_{t}}}$ is a smooth family of bundles over the compact Riemann surface for any $t$ 
(this is just because the Gauss maps are smooth in $t$ ) and furthermore $\overline{J_{\psi_{t}}}$ is a smooth family of differential operators on the sections of the normal bundles.

The same argument as in the proof of Theorem 3.2 in ([1]) shows that the eigenvalues of $\overline{J_{\psi_{t}}}$ depend continuously on $t$. Now the projections of the constant vectors on the normal bundle give rise to $2 n$ independent normal Jacobi fields unless the surface is planar, in which case $\mu^{*}$ cannot be surjective. Therefore $2 n$ is the least possible dimension for the 0 -eigenspace (the space of Jacobi fields). This means that for $|t|$ small enough, 0 -eigensections cannot give rise to negative eigensections of the Jacobi operator.

Therefore index $\left(\overline{J_{\psi_{t}}}\right)=0$ whenever $|t|$ is less than some $t_{0}>0$ as claimed.

Lemma 2.2. For any $t>0, \psi_{t}$ is not holomorphic w.r.t. any compatible complex structure.

Proof. Let us suppose that there exists a compatible complex structure $J$ w.r.t. which $\psi_{t}$ is holomorphic. $J$ has to be of the form $\mathcal{O}^{t} J_{0} \mathcal{O}$ for some $2 n \times 2 n$ orthogonal matrix $\mathcal{O}$. This easily implies that the map $\mathcal{O}\left(\psi_{t}\right)$ is holomorphic w.r.t. $J_{0}$ and therefore is given in real coodinates (up to translations) by

$$
\mathcal{O}\left(\psi_{t}\right)(q)=\operatorname{Re} \int_{q_{0}}^{q}\left(\alpha_{1}, \ldots, \alpha_{n}, i \alpha_{1}, \ldots, i \alpha_{n}\right)
$$

where $\alpha_{j}$ are holomorphic 1-forms on $\Sigma^{*}$ which lie in the span of $d \psi^{1}, \ldots, d \psi^{n}$, which is impossible for $t \neq 0$, because the transformation $\mathcal{O}$ would have changed a non trivial quadratic relation into the trivial one.

\section{Proof of Theorem 1.}

Next result is the key step towards the proof of the existence of holomorphic immersions of finite total curvature with the required special properties. The techniques used in the proof of this result are inspired by ([10]).

Theorem 3. Given a compact Riemann surface of genus two $\Sigma$, a Weierstrass point $p \in \Sigma$ and $n \geq 11$, let $W$ be the space of meromorphic 1-forms on $\Sigma \backslash\{p\}$ which are exact and which have a pole of order at most $n+3$ at $p$. 
Then

1. $\operatorname{dim}(W)=n$;

2. $W$ is base point free;

3. the cup-product map

$$
\tilde{\phi}: W \otimes H^{0}(K) \rightarrow H^{0}\left(K^{2} \otimes[(n+3) p]\right)
$$

is surjective;

4. the cup-product map $\operatorname{Sym}^{2} W \rightarrow H^{0}\left(K^{2} \otimes[2(n+3) p]\right)$ has non trivial kernel.

Proof. Set $m=n+3$ and let us denote by $\phi: H^{0}(K \otimes[m p]) \otimes H^{0}(K) \rightarrow$ $H^{0}\left(K^{2} \otimes[m p]\right)$ the cup product map and by $\omega_{1}, \omega_{2}$ a basis of the space of holomorphic 1-forms on $\Sigma$ (i.e., a basis of $H^{0}(K)$ ).

Now, let now $s_{0}$ be a global holomorphic section of $[m p]$ s.t. $\left(s_{0}\right)=m p$, i.e., $s_{0}$ vanishes to order $m$ at p. We recall (see for example [6], page 136) that if

$$
\mathcal{L}_{m p}=\{\text { meromorphic functions } f \text { on } \Sigma \backslash\{p\} \mid(f)+m p \geq 0\}
$$

then multiplication by $s_{0}$ gives an identification between $\mathcal{L}_{m p}$ and $H^{0}(\mathcal{O}([m p]))$.

We shall keep this identification implicit in what follows. For example, given $h \in \mathcal{L}_{m p}$ we shall identify $d h$ with $d h \otimes s_{0}$ and view $d h$ as an element of $H^{0}(K \otimes[(m+1) p])$.

Before entering into the technical details let us observe that, since $m \geq 3$, by Riemann-Roch

1. $h^{0}([m p])=m-1$;

2. $h^{0}\left(K^{2} \otimes[m p]\right)=m+3$;

3 . if we set $W$ to be the subspace of $H^{0}(K \otimes[m p])$ generated by the meromorphic forms which are exact on $\Sigma \backslash\{p\}$, then $\operatorname{dim}(W)=m-3$ : indeed, by definition, the following sequence (of vector spaces)

$$
0 \rightarrow \mathbb{C} \rightarrow \mathcal{L}_{(m-1) p} \stackrel{d}{\rightarrow} W \rightarrow 0
$$

is exact. But $h^{0}([(m-1) p])=m-2$ and hence the claim follows. 
We now seek a lower bound for $m$ for which all the properties listed in the Theorem hold.

By the base point free pencil trick (see, for example, [6])

$$
\operatorname{ker}(\phi)=\left\{F \omega_{1} \otimes \omega_{2}-F \omega_{2} \otimes \omega_{1} \mid F \in H^{0}(\mathcal{O}[m p])\right\}
$$

and therefore $\phi$ is surjective, since the domain has dimension $2(m+1)$, the target $m+3$, and the kernel $m-1$.

We can write

$$
\operatorname{ker}(\tilde{\phi})=\operatorname{ker}(\phi) \cap\left\{F \omega_{1}=d h_{1}, F \omega_{2}=d h_{2}, h_{i} \in \mathcal{L}_{(m-1) p}\right\} .
$$

To estimate the dimension of this space let us consider the following map

$$
\rho: H^{0}([m p]) \rightarrow H^{1}(\Sigma \backslash\{p\}, \mathbb{C}) \oplus H^{1}(\Sigma \backslash\{p\}, \mathbb{C})
$$

given by $\rho(F)=\left[F \omega_{1}, F \omega_{2}\right]_{\text {DeRham }}$.

Observe that $F \omega_{1} \otimes \omega_{2}-F \omega_{2} \otimes \omega_{1}$ is an element of $\operatorname{ker}(\tilde{\phi})$ if and only if $\rho(F)=0$. Moreover $\rho$ surjective implies that $\tilde{\phi}$ is surjective: in fact if $\rho$ is surjective then $\operatorname{dim} \operatorname{ker}(\rho)=m-9 \geq \operatorname{dim} \operatorname{ker}(\tilde{\phi})$ and therefore $\operatorname{dim} \operatorname{im}(\tilde{\phi}) \geq$ $2 m-6-m+9=m+3=h^{0}\left(K^{2} \otimes[m p]\right)$.

In order to prove that $\rho$ is surjective we need to consider $\rho_{\omega}(F)=$ $[F \omega]_{\text {DeRham }}$ where $\omega \in H^{0}(K)$ is fixed. Since the genus of the surface is two, the zero locus of $\omega, Z(\omega)$, is a divisor of degree 2. Moreover multiplication by $\omega$ gives an isomorphism between $H^{0}(\mathcal{O}([m p]))$ and $H^{0}(K \otimes[m p-Z(\omega)])$. Therefore the surjectivity of $\rho_{\omega}$ is equivalent to the surjectivity of the DeRham-class map $H^{0}(K \otimes[m p-Z(\omega)]) \rightarrow H^{1}(\Sigma \backslash\{p\}, \mathbb{C})$ which depends on the geometry of the divisor $Z(\omega)$.

We need to distinguish three cases:

1. $Z(\omega)=2 p$;

2. $Z(\omega)=2 q, q \neq p$;

3. $Z(\omega)=p+q, q \neq p$

4. $Z(\omega)=q_{1}+q_{2}, q_{1} \neq q_{2}, q_{i} \neq p$.

Since the method used is the same for all the three above cases, we explain it only in case (4): let us set $\mathcal{F}$ the sheaf of holomorphic functions on $\Sigma \backslash\{p\}$, whose differential is a meromorphic form vanishing at $q_{1}$ and $q_{2}$ and with a pole of order at most $m$ at $p$ (note that $\mathcal{F}$ contains naturally tha constant sheaf $\mathbb{C}$ ). We also denote by $\mathcal{G}$ the sheaf of meromorphic forms 
with such zeros and pole, and whose residue at $p$ is zero. We note also that by the residue's theorem $H^{0}(G)=H^{0}(K \otimes[m p-Z(\omega)])$.

We then have the following exact sequence

$$
0 \rightarrow \mathbb{C} \rightarrow \mathcal{F} \rightarrow \mathcal{G} \rightarrow 0
$$

and therefore

$$
\cdots \rightarrow H^{0}(K \otimes[m p-Z(\omega)]) \rightarrow H^{1}(\mathbb{C}) \rightarrow H^{1}(\mathcal{F}) \rightarrow \cdots .
$$

The surjectivity of the De-Rham class map is therefore ensured by the vanishing of $H^{1}(\mathcal{F})$ which is clearly equivalent to the vanishing of $H^{1}\left(\mathcal{O}\left(\left[(m-1) p-2 q_{1}-2 q_{2}\right]\right)\right)$. By Riemann-Roch $H^{1}\left(\mathcal{O}\left(\left[(m-1) p-2 q_{1}-\right.\right.\right.$ $\left.\left.\left.2 q_{2}\right]\right)\right)=0$ whenever $\operatorname{deg}\left(\mathcal{O}\left(\left[(m-1) p-2 q_{1}-2 q_{2}\right]\right)\right)>2$ and therefore for $m>7$. In the same way one can prove directly that in case sufficient conditions for the surjectivity of the DeRham-class map are $m>5$ in case (1) and $m>6$ in the other cases.

We can now go back to study the surjectivity of the map $\rho$. Let us remark that up to now we have not made any particular choice of basis for the space of holomorphic differentials. We now choose $\omega_{1}$ such that $Z\left(\omega_{1}\right)=2 p$.

We claim that the elements of $H^{1}(\Sigma \backslash\{p\}, \mathbb{C}) \oplus H^{1}(\Sigma \backslash\{p\}, \mathbb{C})$ of the form $([0],[\gamma])$ are in the image of $\rho$ for any $[\gamma]$. To see this we choose $F$ in $H^{0}(\mathcal{O}([m p]))$ s.t. $\omega_{1} F=d H$ and define $f=\frac{\omega_{2}}{\omega_{1}}$. By the assumption on $\omega_{1}$ we know that $d H \in H^{0}(K \otimes[(m-2) p])$ and therefore $H \in H^{0}(\mathcal{O}([(m-3) p]))$. The above claim then reduces to asking whether varying $H, f d H$ spans $H^{1}(\Sigma \backslash\{p\}, \mathbb{C})$. To be able to use the above study of the map $\rho_{\omega}$ it is worth observing that $[f d H]=-[H d f]$ and therefore we are asking whether $\rho_{d f}: \mathcal{O}([(m-3) p]) \rightarrow H^{1}(\Sigma \backslash\{p\}, \mathbb{C})$ is surjective.

As before, this depends on the zero locus of $d f$, which is, by standard Riemann surface theory, given by the 5 Weierstrass points, $p_{2}, \ldots, p_{6}$ on $\Sigma$ different from $p$. A sufficient condition for the surjectivity of the map $\rho_{d f}$ is then $H^{1}\left(\mathcal{O}\left(\left[(m-1) p-2 p_{2}-\cdots-2 p_{6}\right]\right)\right)=0$, which happens for $m>13$. Since for such values of $m, \rho_{\omega}$ is also surjective for any $\omega$ the claim implies the surjectivity of the map $\rho$ itself.

We now study condition (3). Let us set $\mathcal{M}$ the sheaf of exact forms in $\mathcal{O}\left(K \otimes\left[(m-1) p-q_{1}-q_{2}\right]\right)$. The base point free condition is well known to be equivalent to the vanishing of $H^{1}(\mathcal{M})$ for any $q_{1} \neq q_{2}$ and both different from $p$ (see, for example, [6]). Denoting by $\mathcal{M}_{q_{1}+q_{2}}$ the skyscraper sheaf obtained by concentrating $\mathcal{M}$ to the divisor $q_{1}+q_{2}$, we can consider the short exact sequence

$$
0 \rightarrow \mathcal{O}\left(\left[(m-1) p-2 q_{1}-2 q_{2}\right]\right) \stackrel{d}{\rightarrow} \mathcal{M} \rightarrow \mathcal{M}_{q_{1}+q_{2}} \rightarrow 0
$$


and the induced long exact one

$$
\cdots \rightarrow H^{1}\left(\mathcal{O}\left(\left[(m-1) p-2 q_{1}-2 q_{2}\right]\right)\right) \rightarrow H^{1}(\mathcal{M}) \rightarrow H^{1}\left(\mathcal{M}_{q_{1}+q_{2}}\right) \cdots .
$$

Since $H^{1}\left(\mathcal{M}_{q_{1}+q_{2}}\right)=0$ the vanishing of $H^{1}(\mathcal{M})$ is therefore ensured by the vanishing of $H^{1}\left(\mathcal{O}\left(\left[(m-1) p-2 q_{1}-2 q_{2}\right]\right)\right)$, which has been proved above.

The last condition to be checked to end the proof of the theorem is (3). We have already observed that $h^{0}\left(K^{2} \otimes[2 m p]\right)=2 m+3$. On the other hand $\operatorname{dim}$ Sym $^{2} W=\frac{(m-3)(m-2)}{2}$. Therefore (3) holds whenever $m^{2}-5 n+6>$ $6+4 m$, i.e. $m>9$, and therefore in our range.

Remark 3.1. On a Riemann surface of genus $g, h^{0}(K)=g, \operatorname{dim}(W)=n$ and $h^{0}\left(K^{2} \otimes[(n+3) p]\right)=n+3 g$. Therefore condition (3) in the above theorem cannot hold for $n=3$. On the other hand it is plausible that $n=4$ is the least dimension for $\mathbb{C}^{n}$ to contain such an affine curve with these properties (but necessarily of genus greater than 4). This would extend further the analogy between the case of compact Riemann surfaces in flat tori and the one of complete minimal surfaces of finite total curvature.

We are now in a position to conclude the proof of our main result. All we need to show is that Theorem 3 implies the existence of holomorphic immersions of $\Sigma \backslash\{p\}$ satisfying all the properties in Theorem 2.

Let $W$ be as in Theorem 3 and $\left\{\eta_{1}, \ldots, \eta_{n}\right\}$ be any basis of $W$.

The map $\psi_{0}: \Sigma \backslash\{p\} \rightarrow \mathbb{C}^{n}$ given by

$$
\psi_{0}(q)=\left(\int_{q_{0}}^{q} \eta^{1}, \ldots, \eta^{n}\right)
$$

is a holomorphic map which is well defined because the $\eta_{j}$ 's are exact.

$\psi_{0}$ is an immersion because the $\eta_{j}$ 's do not have common zeros (condition 3 in Theorem 3).

Condition (2) in Theorem 2 is satisfied by $\psi_{0}$ because of the existence of non trivial kernel in condition 3 in Theorem 3.

We now claim that condition (3) in Theorem 3 implies condition (2) in Theorem 2: we are in fact going to show that $\operatorname{im}\left(\mu^{*}\right)=\operatorname{im}(\tilde{\phi})$. Given a basis $\left\{\omega_{i}\right\}$ of $H^{0}(K)$ by definition we have

1. $\tilde{\phi}\left(a^{j} \eta_{j} \otimes b^{k} \omega_{k}\right)=\left(a^{j} b^{k} \eta_{j} \cdot \omega_{k}\right) \otimes s$, and

2. $\mu\left(c_{1}^{l} \omega_{l}, \ldots, c_{n}^{l} \omega_{l}\right)=\left(c_{1}^{l} \eta_{1} \cdot \omega_{l}+\cdots+c_{n}^{l} \eta_{n} \cdot \omega_{l}\right) \otimes s$. 
Therefore, putting $c_{j}^{l}=a^{j} b^{l}, \operatorname{im}(\tilde{\phi}) \subset \operatorname{im}\left(\mu^{*}\right)$. On the other hand $\left\{\eta_{j} \cdot \omega_{k}\right\}$ is clearly a system of generators for $\operatorname{im}\left(\mu^{*}\right)$, but each of these is obviously in $\operatorname{im}(\tilde{\phi})$ and the claim is proved.

We finally observe that $\psi_{0}$ is an embedding: indeed, as in the classical case of maps into projective spaces $([6])$, one needs to show that $\mathcal{O}([(n-1) p])$ separates points, i.e. $H^{1}\left(\mathcal{O}\left(\left[(n-1) p-q_{1}-q_{2}\right]\right)\right)=0$ for any $q_{1} \neq q_{2}$. By Riemann-Roch this holds for any $n>5$.

Since the embeddedness is an open condition this implies that also $\psi_{t}$, for sufficiently small values of $|t|$, are embeddings.

\section{References.}

[1] C. Arezzo and M.J. Micallef, Minimal surfaces in flat tori, Geom. Funct. Anal., 10 (2000), 679-701.

[2] D. Fischer-Colbrie, On complete minimal surfaces with finite Morse index in three manifolds, Invent. Math., 82 (1985), 121-132.

[3] D. Gieseker, A lattice version of KdV equations, Acta Math., 168 (1992), 219-248.

[4] M. Green, Koszul cohomology and the geometry of projective varieties, J. Differential Geom., 19 (1984), 125-171.

[5] M. Green and R. Lazarsfeld, On the projective normality of complete linear series on an algebraic curve, Invent. Math., 83 (1986), 73-90.

[6] P. Griffiths and J. Harris, Principles of Algebraic Geometry, WileyInter., New York, 1978.

[7] L.P.M. Jorge and W.H. Meeks III, The topology of complete minimal surfaces of finite total Gaussian curvature, Topology, 22 (1983), 203221.

[8] M.J. Micallef, Stable minimal surfaces in Euclidean space, J. Differenrial Geom., 19 (1984), 57-84.

[9] S. Nayatani, On the Morse index of complete minimal surfaces in euclidean space, Osaka J. Math., 27 (1990), 441-451.

[10] G.P. Pirola, Algebraic curves and non rigid minimal surfaces in the euclidean space, Pacific J. Math., 183 (1998), 333-357. 
[11] R. Osserman, A survey of minimal surfaces, Van Nostrand Reinhold, New York, 2nd Edition, 1986.

[12] R. Osserman, Global properties of minimal surfaces in $E^{3}$ and $E^{n}$, Ann. of Math. 80 (1964), 340-364.

[13] J. Simons, Minimal varieties in riemannian manifolds, Ann. of Math., 88 (1968), 62-105.

[14] S.-T. Yau, Open Problems in Geometry, Proc. Symp. Pure Math. A.M.S., 54 (1993), 1-28.

Univ. Di Parma, Italy

E-mail address: arezzo@unipr.it.

UNIV. OF WARWICK, U.K.

E-mail address: mm@maths.warwick.ac.uk

Univ. Di Pavia, Italy

E-mail address: pirola@dragon.ian.pv.cnr.it

Received September 8, 1999. 